\title{
Fatores associados a anemia por deficiência de ferro em crianças pré-escolares brasileiras
}

\author{
Factors associated with iron deficiency anemia \\ in Brazilian preschool children
}

\author{
Carlos A. N. de Almeida1, Rubens G. Ricco², Luiz A. Del Ciampo3, \\ Ana M. Souza ${ }^{4}$, Adriana P. Pinho ${ }^{5}$, José E. Dutra de Oliveira 6
}

\section{Resumo}

Objetivo: Avaliar fatores determinantes de anemia e deficiência de ferro em crianças das duas creches da cidade de Pontal, sudeste do Brasil.

Métodos: Estudo transversal foi realizado avaliando-se 192 crianças com idades entre 12 e 72 meses. Dados pessoais (idade, sexo, uso de ferro medicamentoso, duração do aleitamento materno, tipo de parto, cuidados pré-natais, peso e estatura) e dados sócio-econômicos (número de co-habitantes, escolaridade dos pais e renda per capita familiar) foram obtidos e correlacionados com hemoglobina, receptores de transferrina, ferritina e anemia ferropriva.

Resultados: A idade foi a variável mais afetada pelo estado nutricional de ferro, correlacionando-se com maiores valores de hemoglobina e ferritina e menores valores de receptor de tranferrina, sendo que menos anemia ferropriva foi detectada quanto maior a idade. As outras variáveis estudadas não apresentaram correlação com o estado nutricional de ferro.

Conclusão: Os dados sugerem que as estratégias de controle para essa população de crianças pré-escolares devem ser direcionadas especialmente para aquelas de menor idade.

J Pediatr (Rio J). 2004;80(3):229-34: Anemia, deficiência de ferro, crianças.

\section{Introdução}

A deficiência de ferro é considerada uma das doenças nutricionais de maior prevalência, acometendo mais de dois bilhões de pessoas em todo o mundo ${ }^{1}$. Um grande número de estudos tem demonstrado que em crianças, particularmente aquelas com idade inferior a cinco anos, a anemia por

1. Doutor em Pediatria pela USP; Professor, Universidade de Ribeirão Preto. Diretor do Departamento de Nutrologia Pediátrica da ABRAN.

2. Livre-docente em Pediatria, Fac. de Medicina de Ribeirão Preto, USP.

3. Doutor; Professor de Pediatria, Fac. de Medicina de Ribeirão Preto, USP.

4. Livre-docente, Fac. de Ciências Farmacêuticas de Ribeirão Preto, USP.

5. Mestre em Farmácia pela UNESP e chefe do Laboratório de Análises Clínicas da Universidade de Ribeirão Preto.

6. Professor titular aposentado, Fac. de Medicina de Ribeirão Preto, USP.

Artigo submetido em 28.07.03, aceito em 03.03.04.

\section{Abstract}

Objective: To examine the determining factors of anemia and iron deficiency in children attending two day care centers in the town of Pontal, southeast of Brazil.

Methods: Cross-sectional study was conducted in 192 children aged 12 to 72 months. Personal data (age, sex, use of medicinal iron supplements, duration of breast-feeding, type of delivery, prenatal care, weight, and height), and socioeconomic data (number of coinhabitants, parental schooling, and per capita family income) were obtained and evaluated together with hemoglobin, serum transferrin receptor, ferritin, and iron deficiency anemia.

Results: Age was the variable that most affected iron nutritional status, with higher hemoglobin values, lower transferrin receptor concentrations, higher ferritin values and lower iron deficiency anemia being detected with increasing age. The other studied variables did not show any correlation with iron nutritional status.

Conclusion: The obtained data suggest that control strategies for this preschool population should be especially directed at younger children.

J Pediatr (Rio J). 2004;80(3):229-34: Anemia, iron deficiency, children.

deficiência de ferro (ADF) está relacionada, entre outras alterações, a baixos escores em testes de desenvolvimento mental e de atividade motora, um fator que pode levar a seqüelas irreversíveis, mesmo na presença de tratamento adequado 2,3 .

Apesar da inexistência de estudos nacionais abrangentes, dados regionais têm demonstrado um aumento substancial na prevalência de anemia no Brasil, aumento este observado entre homens e mulheres de todas as idades e níveis socioeconômicos ${ }^{4}$.

A deficiência de ferro e a anemia são causadas por um balanço negativo de ferro no organismo. Apesar da aparente simplicidade do modelo, poucos avanços foram obtidos no que se refere à redução da prevalência de ADF, prova- 
velmente devido ao fato de que esse balanço negativo é o resultado final de uma série de fatores biológicos, sociais, econômicos e culturais que agem sobre a criança. Em contraste com a baixa prevalência mundial de anemia na infância, constatou-se que a prevalência de ADF em crianças que habitam a periferia de grandes cidades norteamericanas é similar à prevalência de ADF observada em países em desenvolvimento ${ }^{5}$. Além disso, vários estudos têm demonstrado uma visível correlação entre deficiência de ferro e anemia e uma série de determinantes, tais como excesso de co-habitantes 6,7 , baixa escolaridade da mãe ${ }^{8}$, alta incidência de parto por cesárea ${ }^{8}$, baixa escolaridade do pai $^{9}$, baixa renda familiar 7,10 e baixas condições socioeconômicas ${ }^{11}$. Assim, é fundamental que se entenda os fatores de riscos de cada população para que se possa oferecer medidas eficazes de prevenção e controle. Nesse contexto, o objetivo deste estudo foi investigar os fatores determinantes da anemia e da deficiência de ferro entre pré-escolares de baixa renda, matriculados em creches públicas em turno integral visando a oferecer um estudo mais detalhado do problema e a produzir um direcionamento para um programa de controle que não seja somente destinado à população estudada, mas a populações com características epidemiológicas similares.

\section{Pacientes e métodos}

O estudo foi realizado na cidade de Pontal, no estado de São Paulo, sudeste do Brasil, no período de $1^{\circ}$ de julho a 31 de dezembro de 1999. A população de estudo consistiu em 225 crianças com idade entre 12 meses completos e 72 meses incompletos, freqüentadoras de duas creches municipais: Creche São Pedro e Centro de Convivência Infantil (CCI). Foram excluídas do estudo 23 crianças que apresentaram doença grave nas últimas duas semanas que antecederam ao estudo; 12 crianças diagnosticadas com doenças crônicas, tais como asma, diabetes, doença do colágeno, e quadros que pudessem interferir no crescimento ou levar a deformidades que poderiam prejudicar as medidas antropométricas (duas crianças); quatro crianças com doenças hematológicas, exceto ADF; oito crianças que já estavam recebendo medicação contendo ferro na data ou nos 30 dias que antecederam a coleta de material e 14 crianças com história de prematuridade ou baixo peso ao nascer. Após a seleção, 192 crianças foram incluídas no estudo. Ambas as creches atendem uma população de baixa renda com pais que trabalham fora, de modo que as crianças permanecem na instituição durante todo o dia.

Os dados pessoais e da família de cada criança foram obtidos em uma única ocasião através de entrevista com os pais ou responsáveis. O peso e a altura foram medidos com instrumentos adequados e calibrados, utilizando-se técnicas precisas e internacionalmente aceitas ${ }^{12}$. Os escores $z$ de peso para altura, peso para idade e altura para idade foram determinados para cada criança de acordo com as recomendações da Organização Mundial da Sáude ${ }^{13,14}$. O sangue venoso foi coletado no mesmo dia que as medidas antropométricas foram realizadas. A hemoglobina foi determinada através de um contador eletrônico de células Coulter T-890 (série 6704465, Nova York, EUA); a ferritina sérica foi medida pelo método imunoenzimático com detecção fluorescente (ELFA) através do equipamento Mini-Vidas BIOMÉRIEUX (série SV 122588, Missouri, EUA); os receptores de transferrina foram determinados através de ensaio imunoenzimático (Quantikine DVD kit, R\&D Systems Minneapolis, EUA), com leitura da absorbância realizada em uma leitora de microplacas 210 ELISA acoplada a um sistema Washer 200-Microwell (Organon Teknica, Áustria); os glóbulos vermelhos foram analisados através do teste de falcização com metabissulfito de sódio para diagnóstico diferencial da anemia falciforme.

As variáveis resposta foram hemoglobina, receptor de transferrina, ferritina e ADF. As crianças com níveis de hemoglobina abaixo do ponto de corte associados a níveis elevados de receptor de transferrina e/ou níveis de ferritina reduzidos foram consideradas portadoras de ADF. As variáveis explanatórias foram divididas em dois grupos:

1) variáveis explanatórias quantitativas: idade, escore $z$ de peso para idade, escore $z$ de peso para altura e escore z de altura para idade;

2) variáveis explanatórias qualitativas ou variáveis quantitativas categorizadas: creches (São Pedro ou CDI), sexo (feminino ou masculino), cuidados pré-natais durante a gestação (sim ou não), número de co-habitantes (menos que quatro ou quatro ou mais), escolaridade dos pais (analfabeto ou alguma instrução), tratamentos prévios com sal de ferro (sim ou não), tipo de parto (normal ou cesárea), aleitamento (exclusivo: somente leite materno, nenhum tipo de sólido ou líquido; predominante: leite materno e outros líquidos como água ou suco de fruta, mas sem alimentação sólida ou leite; complementado: leite materno com alimentação sólida complementar ou outros líquidos) ${ }^{15}$ ou não (menos de 2 meses, de 2 a 6 meses ou mais de 6 meses) e renda per capita familiar mensal (igual/superior a R\$150,00).

Testes de normalidade foram realizados para cada variável resposta (hemoglobina, ferritina e receptor de transferrina) a cada nível das variáveis explanatórias qualitativas nominais, permitindo, dessa forma, a escolha do procedimento estatístico mais adequado para a realização da análise de dados. Tendo a normalidade sido rejeitada por vários testes (ao nível de significância de $5 \%$ ), procedimentos não-paramétricos fora empregados ${ }^{16}$. Os testes de Mann-Whitney e Kruskal-Wallis foram utilizados para avaliar os efeitos das variáveis explanatórias qualitativas/ quantitativas categorizadas sobre as variáveis resposta, com nível de significância estabelecido em 5\%. Os efeitos das variáveis explanatórias quantitativas sobre as variáveis resposta foram avaliados utilizando-se o coeficiente de correlação de Spearman, seguido do teste de associação, com nível de significância estabelecido em $5 \%$.

A análise de regressão logística foi utilizada para determinar a influência das variáveis explanatórias sobre a ADF. Todas as crianças com anemia e, pelo menos, um indicador positivo de deficiência de ferro foram incluídos (hb < $11 \mathrm{mg} / \mathrm{dl}$ e ferritina $<12 \mathrm{ng} / \mathrm{ml}$ e/ou receptor de transferrina $>28 \mathrm{nmol} / \mathrm{ml}$ ). Primeiramente, utilizou-se a análise 
univariada (nível de significância de 5\%) para avaliar o efeito independente de cada variável sobre ADF e, posteriormente, (nível de significância em 20\%) determinar as variáveis para a análise multivariada ${ }^{17}$.

O estudo foi aprovado pelo Comitê de Ética em Pesquisa do Hospital Universitário da Faculdade de Medicina de Ribeirão Preto, Universidade de São Paulo.

\section{Resultados}

A prevalência de anemia (192 crianças avaliadas, hb < $11 \mathrm{mg} / \mathrm{dl}$ ) foi de $62,5 \%$. A prevalência de deficiência de ferro variou de acordo com o indicador estudado, sendo $24,2 \%$ para ferritina ( 132 crianças avaliadas, ferritina $<12 \mathrm{ng} / \mathrm{ml}$ ) e $62,9 \%$ para receptores de transferrina (124 crianças avaliadas, receptor de transferrina $>28 \mathrm{nmol} / \mathrm{ml}$ ). Entre as 119 crianças submetidas a todos os testes laboratoriais, foi comprovada anemia por deficiência de ferro ( $\mathrm{hb}<11 \mathrm{mg} / \mathrm{dl}$ e ferritina $<12 \mathrm{ng} / \mathrm{ml}$ e/ou receptor de transferrina $>28$ $\mathrm{nmol} / \mathrm{ml}$ ) em $51,3 \%$ dos casos.

As Tabelas 1 e 2 apresentam os efeitos das variáveis explanatórias analisadas individualmente sobre as variáveis resposta. O sexo das crianças e as creches por elas freqüentadas não influenciaram os valores da hemoglobina, ferritina ou receptor de transferrina, permitindo a análise do total de meninos e meninas das duas instituições para as variáveis explanatórias restantes. Somente o fator idade mostrou uma correlação positiva com as concentrações de hemoglobina e ferritina e uma correlação negativa com os níveis de receptor de transferrina.

A Tabela 3 apresenta o número de crianças para cada análise ( $n$ ) e o valor p obtido através da análise de regressão logística univariada usada para determinar a influência das variáveis explanatórias sobre a ADF. Um efeito significante $(0,005)$ foi observado somente para idade $(p=0,003)$, com a as crianças menores demonstrando alta probabilidade de apresentarem anemia.

Tabela 1 - Percentis (25, 50 e 75) de hemoglobina, receptor de transferrina e ferritina e valor p em cada nível de variáveis qualitativas

\begin{tabular}{|c|c|c|c|c|c|c|c|c|c|c|c|c|c|c|c|c|}
\hline \multirow[t]{2}{*}{ Variável } & \multirow[t]{2}{*}{ Categoria } & \multicolumn{5}{|c|}{ Hemoglobina } & \multicolumn{5}{|c|}{ Receptor de transferrina } & \multicolumn{5}{|c|}{ Ferritina } \\
\hline & & $\mathbf{n}$ & $\mathbf{P 2 5}$ & P50 & P75 & $\mathbf{p}$ & $\mathbf{n}$ & P25 & P50 & P75 & $\mathbf{p}$ & $\mathbf{n}$ & P25 & P50 & P75 & $\mathbf{p}$ \\
\hline \multirow[t]{2}{*}{ Creche } & São Pedro & 84 & 10,4 & 10,9 & 11,4 & & 55 & 27,3 & 35,0 & 43,1 & & 58 & 10,0 & 24,9 & 41,8 & \\
\hline & $\mathrm{CCI}$ & 108 & 10,2 & 10,9 & 11,6 & 0,90 & 69 & 25,8 & 32,5 & 39,0 & 0,25 & 74 & 12,4 & 20,0 & 32,9 & 0,65 \\
\hline \multirow[t]{2}{*}{ Sexo } & Feminino & 87 & 10,4 & 10,9 & 11,6 & & 60 & 26,0 & 32,5 & 39,6 & & 61 & 12,4 & 25,4 & 37,4 & \\
\hline & Masculino & 105 & 10,3 & 11 & 11,4 & 0,89 & 64 & 26,1 & 34,2 & 41,7 & 0,55 & 71 & 11,6 & 20,5 & 34,1 & 0,54 \\
\hline \multirow{2}{*}{$\begin{array}{l}\text { Cuidados } \\
\text { pré-natais }\end{array}$} & Sim & 143 & 10,3 & 10,9 & 11,4 & & 93 & 25,6 & 32,5 & 40,3 & & 100 & 12,2 & 23,1 & 34,3 & \\
\hline & Não & 13 & 10,6 & 11 & 11,7 & 0,47 & 8 & 26,5 & 36,6 & 42,7 & 0,68 & 8 & 10,2 & 23,0 & 34,6 & 0,77 \\
\hline \multirow{2}{*}{$\begin{array}{l}\text { Número de } \\
\text { co-habitantes }\end{array}$} & Menos de 4 & 26 & 10,1 & 10,9 & 11,3 & & 15 & 23,9 & 39,0 & 55,8 & & 16 & 8,9 & 14,2 & 26,9 & \\
\hline & 4 ou mais & 160 & 10,4 & 11 & 11,6 & 0,17 & 107 & 26,3 & 32,5 & 40,0 & 0,42 & 114 & 12,2 & 23,1 & 36,5 & 0,09 \\
\hline \multirow{2}{*}{$\begin{array}{l}\text { Grau de } \\
\text { escolaridade } \\
\text { do pai }\end{array}$} & Analfabeto & 30 & 10,5 & 11,1 & 11,4 & & 20 & 26,1 & 39,1 & 51,1 & & 19 & 8,9 & 19,6 & 30,6 & \\
\hline & $\begin{array}{l}\text { Alguma } \\
\text { escolaridade }\end{array}$ & 146 & 10,3 & 10,9 & 11,4 & 0,49 & 96 & 26,3 & 33,5 & 39,6 & 0,20 & 105 & 12,2 & 21,2 & 37,4 & 0,17 \\
\hline \multirow{2}{*}{$\begin{array}{l}\text { Grau de } \\
\text { escolaridade } \\
\text { da mãe }\end{array}$} & Analfabeta & 26 & 10,3 & 10,7 & 11,3 & & 16 & 25,2 & 40,1 & 48,5 & & 16 & 7,1 & 15,9 & 30,4 & \\
\hline & $\begin{array}{l}\text { Alguma } \\
\text { escolaridade }\end{array}$ & 160 & 10,4 & 10,9 & 11,6 & 0,28 & 106 & 26,3 & 32,5 & 39,1 & 0,12 & 114 & 12,4 & 21,9 & 35,4 & 0,13 \\
\hline \multirow{2}{*}{$\begin{array}{l}\text { Tratamento } \\
\text { prévio }\end{array}$} & Sim & 96 & 10,2 & 10,9 & 11,4 & & 5 & 26 & 32,5 & 45,6 & & 60 & 6 & 23,3 & 35,2 & \\
\hline & Não & 96 & 10,4 & 11 & 11,5 & 0,30 & 65 & 25,6 & 33,8 & 40,0 & 0,43 & 72 & 12,2 & 20,7 & 33,1 & 0,76 \\
\hline \multirow{2}{*}{$\begin{array}{l}\text { Tipo de } \\
\text { parto }\end{array}$} & Normal & 112 & 10,3 & 11 & 11,6 & & 73 & 25,8 & 33,6 & 41,1 & & 79 & 12,2 & 22,6 & 36,5 & \\
\hline & Cesárea & 70 & 10,4 & 10,9 & 11,4 & 0,52 & 44 & 26,2 & 31,7 & 39,3 & 0,64 & 47 & 11,4 & 20,8 & 32,9 & 0,52 \\
\hline \multirow{3}{*}{$\begin{array}{l}\text { Renda familiar } \\
\text { per capita }\end{array}$} & $U \$ 50,00$ & & & & & & & & & & & & & & & \\
\hline & $\begin{array}{l}\text { ou mais } \\
\text { Inferior a }\end{array}$ & 57 & 10,4 & 10,9 & 11,6 & & 42 & 27,2 & 34,4 & 42,3 & & 42 & 9,8 & 21,0 & 31,7 & \\
\hline & $U \$ 50,00$ & 129 & 10,4 & 11 & 11,4 & 0,71 & 80 & 25,8 & 32,5 & 40,0 & 0,44 & 88 & 12,1 & 22,4 & 37,0 & 0,32 \\
\hline \multirow{3}{*}{$\begin{array}{l}\text { Duração do } \\
\text { aleitamento }\end{array}$} & Menos que $2 \mathrm{~m}$ & 60 & 10,4 & 10,9 & 11,5 & & 43 & 26,4 & 34,2 & 41,1 & & 43 & 11,2 & 19,6 & 34,4 & \\
\hline & 2 a 6 meses & 62 & 9,7 & 10,8 & 11,3 & & 39 & 25,6 & 32,5 & 45,6 & & 42 & 11,6 & 24,8 & 45,3 & \\
\hline & Mais que $6 \mathrm{~m}$ & 66 & 10,7 & 11,1 & 11,7 & 0,08 & 40 & 25,8 & 31,7 & 40,0 & 0,79 & 45 & 12,9 & 22,6 & 34,1 & 0,83 \\
\hline
\end{tabular}


Tabela 2 - Coeficiente de correlação de Spearman e valores p obtidos pelo teste de associação entre as variáveis explanatórias quantitativas e as variáveis resposta hemoglobina, receptor de transferrina e ferritina

\begin{tabular}{lcccccc}
\hline Variável & \multicolumn{2}{c}{ Hemoglobina } & \multicolumn{2}{c}{ Receptor de transferrina } & \multicolumn{2}{c}{ Ferritina } \\
& Coeficiente & $\mathbf{p}$ & Coeficiente & $\mathbf{p}$ & Coeficiente & $\mathbf{p}$ \\
\hline Idade & 0,11 & $<0,01$ & $-0,62$ & $<0,01$ & 0,30 & $<0,01$ \\
Escore z peso por idade & 0,05 & 0,48 & $-0,04$ & 0,67 & $-0,02$ & 0,86 \\
Escore z peso por altura & $-0,04$ & 0,63 & 0,05 & 0,60 & 0 & 0,96 \\
Escore z altura por idade & 0,06 & 0,40 & $-0,01$ & 0,88 & $-0,03$ & 0,71 \\
& & & & & & \\
\hline
\end{tabular}

A Tabela 4 apresenta os valores p obtidos através da análise de regressão logística múltipla. Nesse caso, 163 crianças das quais as observações completas haviam sido obtidas para todas as variáveis com valores $p<0,2$ (Tabela 3). Ao se estabelecer o nível de significância (alfa $=0,005)$, os resultados corroboram aqueles obtidos através da análise univariada, i.e., a idade foi a única variável com efeito significante sobre a ADF.

Tabela 3 - Efeitos das variáveis independentes sobre a anemia por deficiência de ferro determinada pela análise de regressão logística univariada

\begin{tabular}{lcc}
\hline Variável & n & p \\
\hline Creche & 173 & 0,56 \\
Sexo & 173 & 0,75 \\
Cuidado pré-natal & 139 & 0,44 \\
Número de co-habitantes & 167 & $0,08 *$ \\
Grau de instrução do pai & 158 & 0,98 \\
Grau de instrução da mãe & 167 & 0,23 \\
Tratamento prévio & 173 & 0,89 \\
Tipo de parto & 163 & 0,72 \\
Renda familiar per capita & 167 & 0,79 \\
Duração do aleitamento (2 a 6 meses) & 169 & $0,08 *$ \\
Duração do aleitamento (mais que 6 meses) & 169 & $0,07 *$ \\
Idade & 173 & $0,03+$ \\
Escore z peso para idade & 173 & 0,78 \\
Escore z peso para altura & 173 & 0,24 \\
Escore z altura para idade & 173 & 0,34 \\
\end{tabular}

${ }^{*} p<0,20 \quad+p<0,05$
Tabela 4 - Efeito das diferentes variáveis sobre a anemia por deficiência de ferro determinada pela analise de regressão logística múltipla

\begin{tabular}{ll}
\hline Variável & p \\
\hline Número de co-habitantes & 0,12 \\
Duração do aleitamento (2 a 6 meses) & 0,32 \\
Duração do aleitamento (mais que 6 meses) & 0,06 \\
Idade & $0,04 *$ \\
\hline
\end{tabular}

${ }^{*} p<0,05$

\section{Discussão}

A determinação dos fatores que influenciam a ocorrência e a manutenção da anemia e da deficiência de ferro em uma população é fundamental para a implementação de medidas de controle. Embora este estudo tenha sido conduzido em uma população específica, é possível que os dados obtidos sejam extrapolados para países em desenvolvimento e para subpopulações de baixa renda em países desenvolvidos, sob condições socioeconômicas similares.

Entre os fatores analisados, somente a idade exerceu influência sobre todos os indicadores do estado nutricional de ferro estudados. Quanto às concentrações de hemoglobina, a mesma tendência foi observada em outros estudos desenvolvidos sob diferentes condições ${ }^{18-21}$. Nicklas et al. ${ }^{22}$, ao estudarem pré-escolares no Haiti, observaram a existência de uma correlação entre hemoglobina e idade e sugeriram que com o aumento da idade as crianças são capazes de consumir uma dieta mais variada incrementando, dessa forma, seus indicadores de hemoglobina. A correlação observada entre a idade e os níveis dos receptores de transferrina, com a redução desses níveis associada ao aumento da idade, demonstra um aumento da disponibilidade de ferro em crianças mais velhas. Quanto à ferriti- 
na, Nicklas et al. 22 também observaram um aumento dos valores de ferritina associada ao aumento de idade das crianças haitianas entre 2 a 5 anos. A correlação entre idade e ADF nos permite supor que crianças mais jovens apresentam maior probabilidade de manifestarem o espectro total da deficiência de ferro, variando de um declínio nas reservas de ferro à anemia por deficiência de ferro. Por outro lado, é fundamental que se continue a discussão sobre os valores de referência e a necessidade eventual de ajustá-los à idade; um requisito que só poderá ser satisfeito com estudos que determinem correlações clínico-laboratoriais.

Quanto às demais variáveis estudadas, o tratamento prévio com ferro não influenciou o estado de ferro, embora estivesse presente em metade das crianças. Baseado em um fato já conhecido de que a recuperação dos indicadores de deficiência segue a ordem inversa da depleção, i.e., a recuperação da hemoglobina ocorre primeiro e a recuperação da ferritina por último, era de se esperar que a ferroterapia prévia levasse a maiores concentrações de hemoglobina, mas esse não foi o caso. Mahu et al. ${ }^{23}$, ao estudarem 384 crianças de Réunion com idade inferior a seis anos, também não observaram nenhum efeito da ferroterapia prévia sobre os valores de hemoglobina. Roughead \& Hunt ${ }^{24}$ observaram que a suplementação com ferro medicamentoso reduz a absorção do ferro não-heme da dieta dos adultos, um fato que em muitos casos nulifica o efeito benéfico do medicamento sobre as reservas de ferro. Por outro lado, essa constatação pode indicar que a ferroterapia oral, na forma que é comumente realizada no Brasil, i.e., doses diárias de sal de ferro, não é suficiente para combater a anemia e a deficiência de ferro. Na verdade, muitos autores têm demonstrado que a alta incidência de efeitos colaterais quase sempre leva à baixa aceitação desse tipo de tratamento 25,26 .

Estudos desenvolvidos em diferentes partes do mundo têm demonstrado resultados conflitantes no que se refere à correlação entre anemia e indicadores antropométricos. De Almeida et al. ${ }^{27}$ não observaram qualquer influência dos escores $z$ das medidas antropométricas sobre os valores de hemoglobina, embora deva ser salientado que a população de estudo apresentava baixas prevalências de subnutrição e obesidade. Em contraste, O'Donnell et al. ${ }^{8}$, ao estudarem crianças pré-escolares carentes de Buenos Aires, observaram uma correlação entre anemia e baixo escore $z$ para altura por idade. Na verdade, não foi observada nenhuma correlação entre anemia e indicadores antropométricos entre populações com estado nutricional adequado7,23.

De acordo com o modelo corrente, a ferritina, a reserva de ferro do organismo, é o primeiro compartimento a sofrer depleção no caso de balanço de ferro negativo 28 . Portanto, esperar-se-ia que os determinantes avaliados neste estudo exercessem maior efeito nessa variável do que em outros parâmetros estudados, mas esse não foi o caso. Outros autores já observaram correlações entre ferritina e diferentes variáveis, tais como peso corporal ${ }^{29}$, baixa classe soci$\mathrm{al}^{30}$ e sexo. ${ }^{31} \mathrm{~A}$ avaliação do efeito do tempo de uso do leite materno sobre a ferritina seria de especial interesse. No presente estudo, não foi observada diferença entre os três grupos estudados. Innis et al. 32 concluíram que o aleitamento resultou em baixos níveis de ferritina em crianças canadenses independente de sua duração. Hokama33 observou níveis baixos de ferritina em crianças japonesas que receberam exclusivamente leite materno em comparação com aquelas que receberam leite de outras fontes como complemento. Um grande número de estudos, como o de Saarinen ${ }^{34}$ na Turquia, Pastel et al. ${ }^{35}$ no Peru e Franco et al. ${ }^{36}$ no Chile, demonstraram que [mesmo] o aleitamento exclusivo é capaz de manter reservas adequadas de ferro, representado por níveis satisfatórios de ferritina, até, pelo menos, seis meses de idade. Os dados obtidos do presente estudo mostram a inexistência de correlação entre estado nutricional de ferro e aleitamento de curto ou longo prazo para a faixa etária estudada. Várias limitações devem ser levadas em consideração ao interpretarmos esse resultado. A principal foi o fato de que, no grupo das crianças amamentadas, não discriminamos entre aleitamento exclusivo e não-exclusivo; consideramos unicamente o efeito do aleitamento como uma boa fonte de ferro para a criança. Outro possível viés foi a análise das faixas etárias como um todo, o que pode ter diluído o efeito da variável, já que crianças mais jovens, recém-desmamadas foram analisadas juntamente com outras crianças bem mais velhas, submetidas a diferentes tipos de dietas.

Contrastando com outras doenças de carência, cujas estratégias de controle sempre consistem em intervenção do contexto socioeconômico, os dados obtidos indicam abordagens mais objetivas e factíveis para a população estudada. Esforços e recursos financeiros devem ser especificamente concentrados na implementação de medidas que visem ao incremento do fornecimento de ferro, especialmente para crianças mais jovens, através de medicação e fortificação de alimentos consumidos habitualmente. Similarmente, outros países, especialmente aqueles em desenvolvimento que conseguiram controlar a desnutrição proteico-calórica, mas ainda apresentam alta prevalência de anemia, podem lançar mão de estratégias semelhantes para o controle desse problema mundial de saúde pública.

\section{Referências}

1. Layrisse M, Cháves JF, Mendez-Castellano H, Bosch V, Tropper E, Bastardo B, et al. Early response to the effect of iron fortification in the Venezuelan population. Am J Clin Nutr. 1996;64:903-7.

2. Osky FA. The nonhematologic manifestations of iron deficiency. Am J Dis Child. 1979;133:315-22.

3. Lozoff B, Wolf AW, Jimenez E. Iron-deficiency anemia and infant development: effects of extended oral iron therapy. J Pediatr. 1996;129:382-9.

4. Monteiro CA, Szarfarc SC, Mondini L. Tendência secular da anemia na infância na cidade de São Paulo (1984-1996). Rev Saúde Pública. 2000;34:62-72.

5. Booth IW, Aukett MA. Iron deficiency anemia in infancy and early childhood. Arch Dis Child. 1997;76:549-54.

6. Czajka-Narins DM, Haddy TB, Kallen DJ. Nutrition and social correlates in iron deficiency anemia. Am J Clin Nutr. 1978;31:955-60.

7. Neuman NA, Tanaka OU, Szarfarc SC, Guimarães PRV, Victora CG. Prevalência de fatores de risco para anemia no Sul do Brasil. Rev Saúde Pública. 2000;34:56-63. 
8. O'Donnell A, Carmuega ES, Durán P. Preventing iron deficiency in infants and preschool children in Argentina. Nutr Rev. 1997; 55:189-94.

9. Nestel P, Melara A, Rosado J, Mora JO. Vitamin A deficiency and anemia among children 12-71 months old in Honduras. Rev Panam Salud Publica. 1999;6:34-43.

10. Romani SAM, Lira PIC, Batista Filho M, Sequeira LÃS, Freitas CLC. Anemias em pré-escolares: diagnóstico, tratamento e avaliação em Recife-PE, Brasil. Arch Latinoam Nutr. 1991;41:159-67.

11. Marx JJM. Iron deficiency in developed countries: prevalence, influence of lifestyle factors and hazards of prevention. Eur J Clin Nutr. 1997;51:491-4.

12. Cameron N. The measurement of human growth. London: Croom-Helm; 1984.

13. WHO Working Group on Infant Growth. An evaluation of infant growth: the use and interpretation of anthropometry in infants. Bull WHO. 1995;73:165-74.

14. Onis M, Habicht JP. Anthropometric reference data for international use: recommendations from a World Health Organization Expert Committee. Am J Clin Nutr. 1996;64:650-8.

15. WHO. Indicators for assessing breastfeeding practices. Geneva: WHO; 1991.

16. Hollander M, Wolfe DA. Nonparametric Statistical Methods. 2nd ed. New York: John Wiley \& Sons; 1973.

17. Hosmer DW, Lemeshow S. Applied Logistic Regression. New York: John Wiley \& Sons; 1989.

18. Hossain MM, Bakir M, Pugh RNH, Sheekh-Hussen M, Ishaq SAB, Berg DB, et al. The prevalence and correlates of anemia among young children and women of childbearing age in Al Ain, United Arab Emirates. Ann Trop Paed. 1995;15:227-35.

19. Assis AMO, Santos LMP, Martins MC, Araújo MPN, Amorin DQ, Morris SS, et al. Distribuição da anemia em pré-escolares do semi-árido da Bahia. Cad Saúde Pública (Rio de Janeiro). 1997; 13:237-43

20. Cornet M, Hesran JY, Fievet N, Cot M, Personne P, Gounoue R, et al. Prevalence of and risk factors for anemia in young children in Southern Cameroon. Am J Trop Med Hyg. 1998;58:606-11.

21. Giebel HN, Suleymanova D, Evans GW. Anemia in young children of the Muynak District of Karakalpakistan, Uzbekistan: prevalence, type and correlates. Am J Public Health. 1998;88:805-7.

22. Nicklas TA, Kuvibidila S, Gatewood LC, Metzinger AB, Frempong $\mathrm{KO}$. Prevalence of anemia and iron deficiency in urban Haitian children two to five years of age. J Trop Pediatr. 1998;44:133-8.

23. Mahu JL, Valteau D, Suquet JP, Meskens C, Saly JC, Lebas J. Prevalence and etiology of anemia in Reunion: comparative study of hematological, biochemical and anthropometric parameters, parasitic and clinical status of anemic and nonanemic children. Nutr Res. 1988;8:733-44.
24. Roughead ZK, Hunt JR. Adaptation in iron absorption: iron supplementation reduces nonheme-iron but not heme-iron absorption from food. Am J Clin Nutr. 2000;72:982-9.

25. Cardoso MA, Penedo MVC. Intervenções nutricionais na anemia. Cad Saúde Pública (Rio de Janeiro). 1994;10:231-40.

26. Batista Filho M, Ferreira LOC. Prevenção e tratamento da anemia nutricional ferropriva: novos enfoques e perspectivas. Cad Saúde Pública (Rio de Janeiro). 1996;12:411-5.

27. De Almeida CAN, Ricco RG, Del Ciampo LA, Souza AM, Dutra de Oliveira JE. Growth and hematological studies on Brazilian children of low socioeconomic level. Arch Latinoam Nutr. $2001 ; 51: 230-5$.

28. Expert Scientific Working Group. Summary of a report on assessment of the iron nutritional status of the United States population. Am J Clin Nutr. 1985;42:1318-30.

29. Dommergues JP, Breton MP, Ducot B, Yvart J, Rossignol C, Tchernia G. Iron deficiency in infants. Study of risk factors. Arch Fr Pediatr. 1984;41:623-7.

30. Mesanza PL, Martinez EO, Altuna MS, Frances MAP, Dolora MJU, Jimenez JLL. Anemia and depletion of iron reserves in healthy 12 month old infants. An Esp Pediatr. 1992;37:24-8.

31. Wharf SG, Fox TE, Fairweather-Tait SJ, Cook JD. Factors affecting iron stores in infants 4-18 months of age. Eur J Clin Nutr. 1997;51:504-9.

32. Innis SM, Nelson CM, Wadsworth LD, MacLaren IA, Lwanga D. Incidence of iron-deficiency anaemia and depleted iron stores among nine-month-old infants in Vancouver, Canada. Rev Canad Sant Pub. 1997;88:80-4.

33. Hokama T. Levels of serum ferritin and total iron among infants with different feeding regimens. Acta Paediatr Jpn. 1993;35:298-301.

34. Saarinen UM. Need for iron supplementation in infants on prolonged breast feeding. J Pediatr. 1978;93:177-80.

35. Pastel RA, Howanitz PJ, Osky FA. Iron sufficiency with prolonged exclusive breast-feeding in Peruvian infants. Clin Pediatr. $1981 ; 20: 625-6$.

36. Franco E, Hertrampf E, Rodriguez E, Illanes JC, Palácios L, Llaguno $S$, et al. Iron nutrition in Mapuche infants fed with human milk (2d phase). Rev Chil Pediatr. 1990;61:248-52.

Correspondência:

Carlos Alberto Nogueira de Almeida

Rua Tamoios, 262/154

CEP 14020-700 - Ribeirão Preto, SP

Tel./fax: (16) 621.8130

E-mail: carlosnogueira@directnet.com.br 